\title{
Discrete Wavelet Transform and Bird Swarm Optimized Bayesian Multimodal Medical Image Fusion
}

\author{
${ }^{* 1}$ Jayant Bhardwaj, ${ }^{2}$ Abhijit Nayak \\ ${ }^{1}$ University School of Information, Communication and Technology and Department of ECE, Bhagwan \\ Parshuram Institute of Technology, Guru Gobind Singh Indraprastha University, New Delhi, India \\ ${ }^{2}$ Department of Applied Physics, Bhagwan Parshuram Institute of Technology, Delhi, India \\ Email: jayantbhardwaj@ieee.org,nayakabhijit@gmail.com
}

Received: 11th November 2019, Accepted: 08th January 2020, Published: 29th February 2020

\begin{abstract}
A novel Bayesian image fusion scheme using bird swarm optimization algorithm (BSA) is being proposed here. The medical Image fusion is progressed using the MRI brain images taken from the BRATS database and the source images of different modalities are fused effectively to present an information rich fused image. The source images are subjected to the Haar discrete wavelet transform (DWT) and the Bayesian fusion is performed using the Bayesian parameter, which is determined optimally using the BSA optimization. The analysis reveals that the method outperformed the three existing methods of fusion that is nonsubsampled contourlet transform (NSCT), cascaded static wavelet (SWT) and NSCT , that is (SWT-NSCT) and Holoentropy and SP-Whale Optimization method (HW Fusion) with improved values of mutual information(1.4764), peak signal-to-noise ratio (37.2114) and root mean square error (9.9341).
\end{abstract}

Keywords

Image Fusion; Bird Swarm; MRI; Haar Wavelet; Bayesian

\section{Introduction}

Image fusion (IF) aims to maximize the information in an image through the collection of the effective features ensured by different modalities[1]. Medical IF addresses the raising demands in the clinical application and it enables the easy access for extracting enormous significant information necessary for disease diagnosis with minimal storage cost [2]. Wavelet transform based different image fusion schemes are quite popular from years. There is an exponential growth of the non-zero coefficients when the wavelet represent the multi-dimensional features, like contours and there is an increase in the non-zero coefficients and are not disregarded for their large amplitude, assuring the loss in the directional sensitivity[3]. There are a number of algorithms of IF using the wavelet transform as they hold a number of advantages to capture the feature data from the available source images. The primary intention of this research is to design and develop a technique for multimodal image fusion based on Bayesian fusion approach. Patil Hanmant Venkatrao et al.[2] developed, holo entropy and SP-Whale optimisation algorithm. The performance of the method was high, but failed considering the Bayesian theory that was found to be the point of improvement in IF. Vikrant Bhateja et al. [3] modelled the stationary wavelet transform (SWT) and non sub-sampled contourlet transform (NSCT)[3],[4],[5], which minimized the redundancy and rendered enhanced performance due to the features. Moreover, there was no down-sampling for the method compared with SWT[3] and so, there was no degradation irrespective to the shift in the source images. However, the drawback was regarding the non-directionality of the decomposed coefficients. Initially, two image data sets from BRATS database[11],[12] with different modalities of MRI images, like T1, T2, T1C, and FLAIR are taken for image fusion as shown in Fig. 1 . The input image taken for fusion is subjected to the Haar discrete wavelet transform(DWT)[6] that converts the input images into four frequency sub bands that is low-high (LH),low-low( LL), high-high(HH) and high-low(HL)[7],[8]. This is called subband coding and employs filter $h$ and $g$ as shown in Fig. 2.These four wavelet coefficients so obtained of two input images are fed to Bayesian fusion approach, which fuses the Haar wavelet coefficients of both the images using Bayesian parameters[9]. The optimal parameters for the Bayesian fusion approach is computed using the BSA, which is a newly developed fusion approach[10]. Then, the resulting image is given to inverse Haar wavelet transform that generates the fused image.
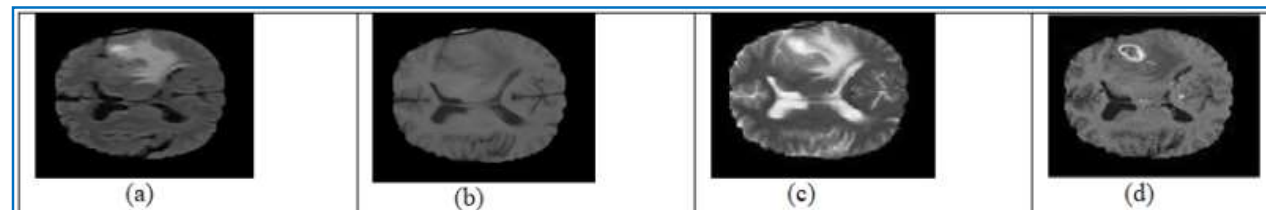

Fig 1: Multimodal Image Data Set (a)Flair Image (b)T1 Image(c) T2 Image (d)T1C Image 


\section{Methodology}

The proposed fusion scheme comprises of images decomposition using Haar discrete wavelet transform (DWT) and fusion rule using a popular probabilistic estimator named Bayesian model . The proposed scheme is shown in Fig.2 .

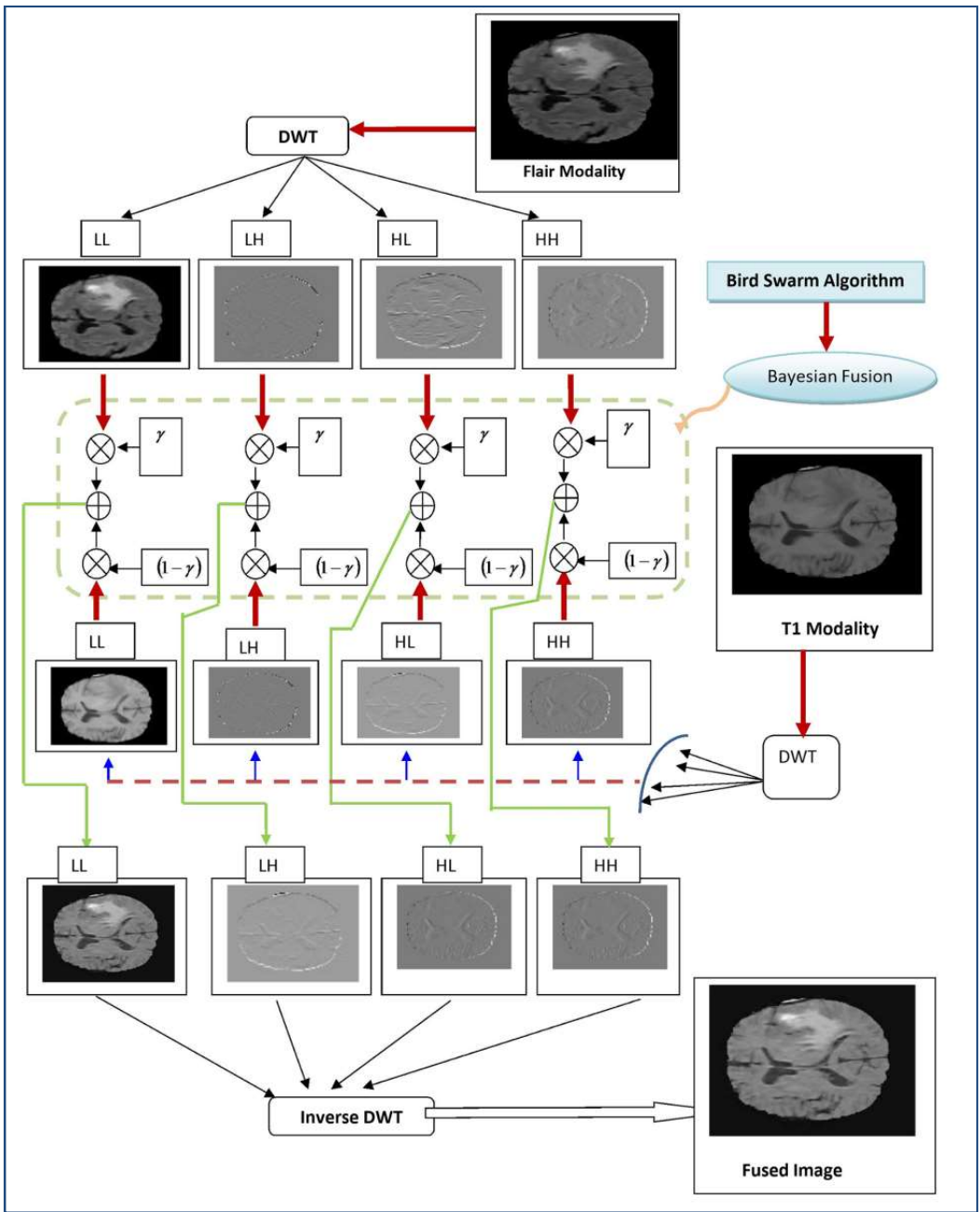

Fig 2: Proposed DWT and BSA-Bayesian Fusion Scheme

\section{Image Decomposition using Haar Discrete Wavelet Transform}

The Haar wavelet transform is DWT known for temporal resolution . For the input image listed in $2^{u}$ numbers, the input pairs of Haar wavelet are generated, difference is saved, and calculates the sum, which is repeated iteratively until the sums are paired to ensure the next scale. At last, $2^{u}-1$ differences and a final sum is derived. The orthonormal transform of Haar wavelet is represented as,

$X=N I_{j} N^{T}$

where, $N$ refers to the Haar transformation matrix of dimension $(B \times B), I_{j}$ is the input image matrix of size $(B \times B)$, and $X$ is the Haar transform of size $(B \times B)$, which possess the Haar functions that are defined in the interval $T \in 0,1$ for $\ell=0,1, \ldots, B-1$ and $B=2^{u}$. For the generation of $N, \ell$ is given as,

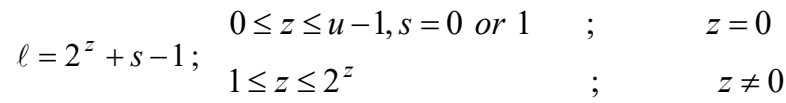

The Haar basis functions are defined in [6]. The operation of Haar wavelet transform when applied to an input image $I_{j}$ with $(B \times B)$ samples are shown as: The individual row corresponding to the input image is filtered via the filters, low-pass and high-pass filters, and the output is down-sampled to obtain the sub-images, $\mathrm{L}$ and $\mathrm{H}$ images, which present the high and low frequency images. These two images are again filtered using low- and high-pass band filters and down sampled to generate the sub-images, LL, HL, LH, and HH that are combined to 
generate the original image of same $(B \times B)$ samples. LL specifies the low-frequency component, whereas the sub-bands HL, LH, and HH correspond to the high-frequency components[7],[8] as shown in Fig 3.. These subbands are employed for medical imaging in which the images with complementary details are combined as an image to preserve the sensitive details to enable diagnosis.

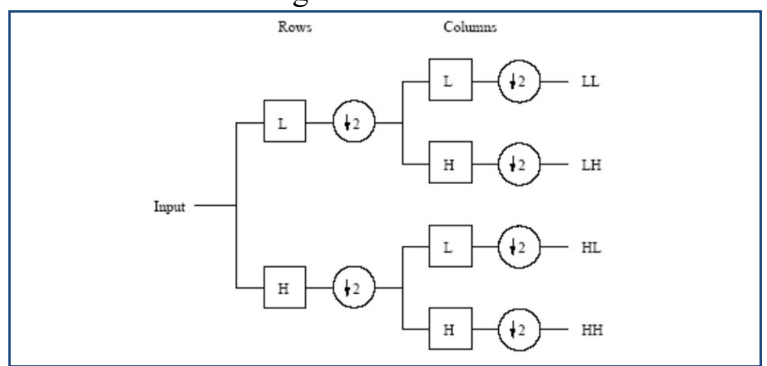

Fig 3: One Stage in DWT Image Decomposition

Bayesian Fusion

The sub images of different frequency bands obtained as wavelet coefficients that is LL,LH,HL,LL are extracted from both source images $\mathrm{I}_{1}(\mathrm{x}, \mathrm{y})$ and $\mathrm{I}_{2}(\mathrm{x}, \mathrm{y})$ with two level decomposition by haar DWT These sub-images are employed for IF using an eminent probabilistic model called Bayesian modal[9]. The individual pixels in the sub images of both source images are fused for which the Bayesian parameter $\gamma$ is employed which is determined optimally using the BSA[10]. The fusion of the low sub-band corresponding to $\mathrm{I}_{1}(\mathrm{x}, \mathrm{y})$ with low sub-band of $\mathrm{I}_{2}(\mathrm{x}, \mathrm{y})$ is done using the Bayesian parameter $\gamma$. The Bayesian factor is tuned optimally using the BSA in such a way that the value of the factor renders the effective fusion in order to acquire good quality[9],[10].

Bayesian model optimization (BSA-Bayesian)

The BSA is based on the social interactions and behaviours of birds, which includes the foraging, vigilance, and flight behaviours[10]. Another interesting phenomenon is regarding the foraging behaviour such that during the foraging mechanism, the bird keeps in record the previous best experience acquired along with the best experience of the swarm in order to search their food in the present. Likewise, the information is carried to the entire swarm and speaking about the vigilance, the bird moves to the centre of the swarm, which could be affected with the interference occurring during the competition in the swarm[10]. The most significant mechanism is birds shift between the places during production and scrounging and the birds with highest reserve, lowest reserve, and others are referred as producer, scrounger, and the rest of the birds choose randomly between producing and scrounging. The producers play a major role of searching food, whereas the scroungers follow the producers for their food. The foraging behaviour of the birds is modelled as follows[10].

Foraging behaviour: the foraging behaviour is given as,

$A_{b, d}^{\tau+1}=A_{b, d}^{\tau}+\left(P_{b, d}^{\tau}-A_{b, d}^{\tau}\right) \times p_{1} \times \operatorname{rand}(0,1)+\left(G_{d}-A_{b, d}^{\tau}\right) \times p_{2} \times \operatorname{rand}(0,1)$

where, $b$ refers to the total birds in the population and it is given as, $b \in 1, \ldots, m$ and $m$ stands for the total number of birds in the population. The dimensional space is denoted as, $d$, which is given as, $d \in 1, \ldots, S$ and $\tau$ specifies the time-step. The previous best position of the $b^{\text {th }}$ bird in $d^{\text {th }}$ dimension is notated as, $P_{b, d}^{\tau}$ and the previous best position of the bird swarm is denoted as, $G_{d}$. The position of the $b^{\text {th }}$ bird in $d^{\text {th }}$ dimension is notated as, $A_{b, d}^{\tau}$, rand $(0,1)$ specifies the independent members distributed in $(0,1)$ with $p_{1}$ and $p_{2}$ being the positive numbers, presenting the cognitive as well as social accelerated parameters. Whenever the uniform random number $(0,1)$ lies below the constant const $(0,1)$, the bird continues foraging for discovering the food or else the birds continue with the vigilance behaviour in the swarm[10].

Vigilance behaviour: The vigilance behaviour of the birds is modelled as,

$A_{b, d}^{\tau+1}=A_{b, d}^{\tau}+J_{1}\left(\mu_{d}-A_{b, d}^{\tau}\right) \times \operatorname{rand}(0,1)+J_{2}\left(P_{i d}-A_{b, d}^{\tau}\right) \times \operatorname{rand}(-1,1)$

$J_{1}=r_{1} \times \exp \left(-\frac{F_{b}{ }^{p}}{\sum F+\beta} \times m\right) ; i \neq b ; 1 \leq i \leq m$

$J_{2}=r_{2} \times \exp \left[\left(\frac{F_{b}^{p}-F_{i}^{p}}{\left|F_{i}^{p}-F_{i b}^{p}+\beta\right|}\right) \frac{n \times F_{i}^{p}}{\sum F+\beta}\right]$ 
where, $F_{b}{ }^{p}$ refers to the value of best fitness of $i^{\text {th }}$ bird, $\sum F$ refers to the sum of the swarm's best fitness measure, $\beta$ signifies the smallest constant, and $\mu_{d}$ is the mean of $d^{\text {th }}$ element of average position of whole swarm. Let $r_{1}$ and $r_{2}$ are the positive constants varying between 0 and 2 and $m$ specify the total birds in the population. During the vigilance, the bird moves to the centre in such a way that $J_{1}$ and $\operatorname{rand}(0,1)$ is not more than one and at the same time, the direct impact of the interference is measure with $J_{2}$. Whenever the fitness of the random $i^{\text {th }}$ bird is better than the $b^{\text {th }}$ bird, the $J_{2}$ is said to be greater than $r_{2}$, which implies that the bird suffers from interference and more specifically, $b^{\text {th }}$ bird faces more interference compared with $b^{\text {th }}$ bird. The above equation (4) describes the standard equation of the BSA in the vigilance behaviour[10]. The birds fly to places because of the threats or foraging in-search of food. The producers search for food, while the other birds follow the producers for food. The flight behaviour is foreseen as[10],

$A_{b, d}^{\tau+1}=A_{b, d}^{\tau}+\operatorname{rand} g(0,1) \times A_{b, d}^{\tau}$

$A_{b, d}^{\tau+1}=A_{b, d}^{\tau}+\left(A_{i, d}^{\tau}-A_{b, d}^{\tau}\right) \times l \times \operatorname{rand}(0,1)$

rand $g(0,1)$ refers to the Gaussian distributed random number with $\mu=0$ and $S D=1 ; 1 \leq i \leq n ; i \neq b$, and $l \in 2$. The effective Bayesian training model[9] is developed using the optimization and the class label is derived using the posterior probability and the class label that acquires the maximal value of the posterior probability is set to the data. Thus, the class value derived using the posterior probability is employed as the value of $\gamma$ during the medical IF, which enables the high quality image and optimal fusion [9][10].

\section{Results and Discussion}

The experimentation is performed on Intel i5 ,3.0 Ghz CPU based Computer and with Matlab R2018 software using brain MRI images from the BRATS 2018 database [11],[12].PSNR is a measure of the quality of the fused medical Image, which is computed using the fused original image and the source images. RMSE is the measure of the error, which measures the deviation in the fused image compared with the desired output. The Fig. 4(a)4(c)shows the performance of proposed method of fusion(BSA-Bayesian) for different modalities for different values of iterations $(50,100,150$ and 200)through the observed values of assessment parameters that is MI,PSNR and RMSE. Table 1 describes the comparative values of MI,PSNR and RMSE for fusion of Flair and T1(Image set-1) and Flair and T1C modalities(Image set-2) respectively with proposed strategy (BSA-Bayesian) ,HW Fusion[2] ,SWT-NSCT[3] and NSCT[4],[5] as shown in Fig.5(a)-5(c). The results may varies for different image sets. This can be analysed by Table 1 and Table 2 .

Table 1: Comparative Analysis for Image - Set 1(Flair and T1)

\begin{tabular}{|c|c|c|c|c|}
\hline Metrics & $\begin{array}{c}\text { Wavelet + HW } \\
\text { Fusion }\end{array}$ & SWT + NSCT & NSCT & $\begin{array}{c}\text { Proposed BSA- } \\
\text { Bayesian Fusion }\end{array}$ \\
\hline Mutual Information & 1.3673 & 1.3684 & 1.3961 & $\mathbf{1 . 4 7 6 4}$ \\
\hline PSNR (in dB) & 33.8944 & 33.8848 & 32.8904 & $\mathbf{3 7 . 2 1 1 4}$ \\
\hline RMSE & 11.3101 & 11.5281 & 10.5409 & $\mathbf{9 . 9 3 4 1}$ \\
\hline
\end{tabular}

Table 2: Comparative Analysis for Image Set -2(Flair and T1C)

\begin{tabular}{|c|c|c|c|c|}
\hline Metrics & $\begin{array}{c}\text { Wavelet + HW } \\
\text { Fusion }\end{array}$ & SWT + NSCT & NSCT & $\begin{array}{c}\text { Proposed BSA- } \\
\text { Bayesian Fusion }\end{array}$ \\
\hline Mutual Information & 1.3873 & 1.3784 & 1.4961 & $\mathbf{1 . 4 8 6 4}$ \\
\hline PSNR (in dB) & 33.9944 & 33.9848 & 32.9904 & $\mathbf{3 7 . 3 1 1 4}$ \\
\hline RMSE & 11.3301 & 11.5481 & 10.5509 & $\mathbf{9 . 9 4 4 1}$ \\
\hline
\end{tabular}




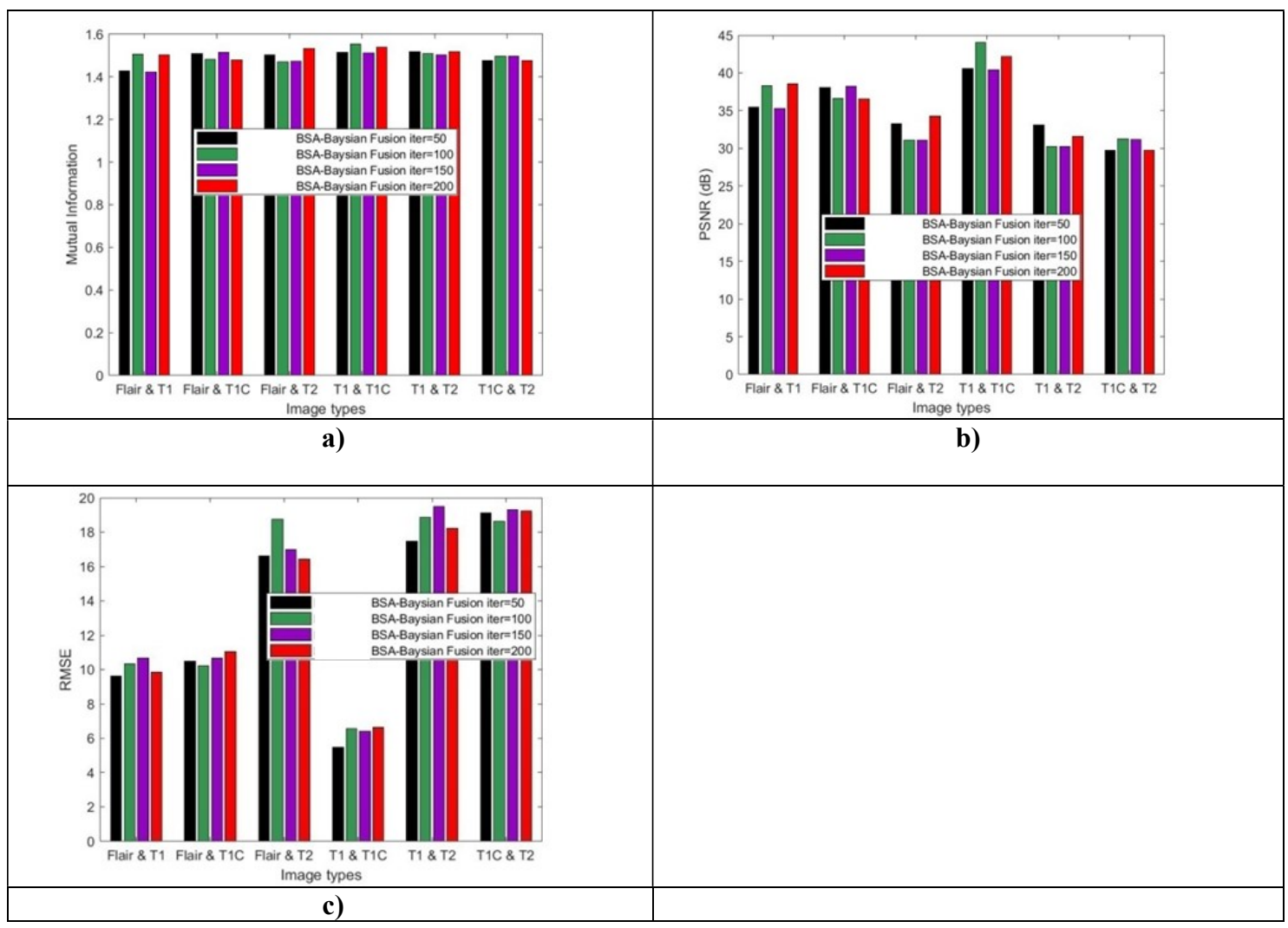

Fig 4: Performance Analysis of Proposed Method, a) Mutual Information, b) PSNR, c) RMSE

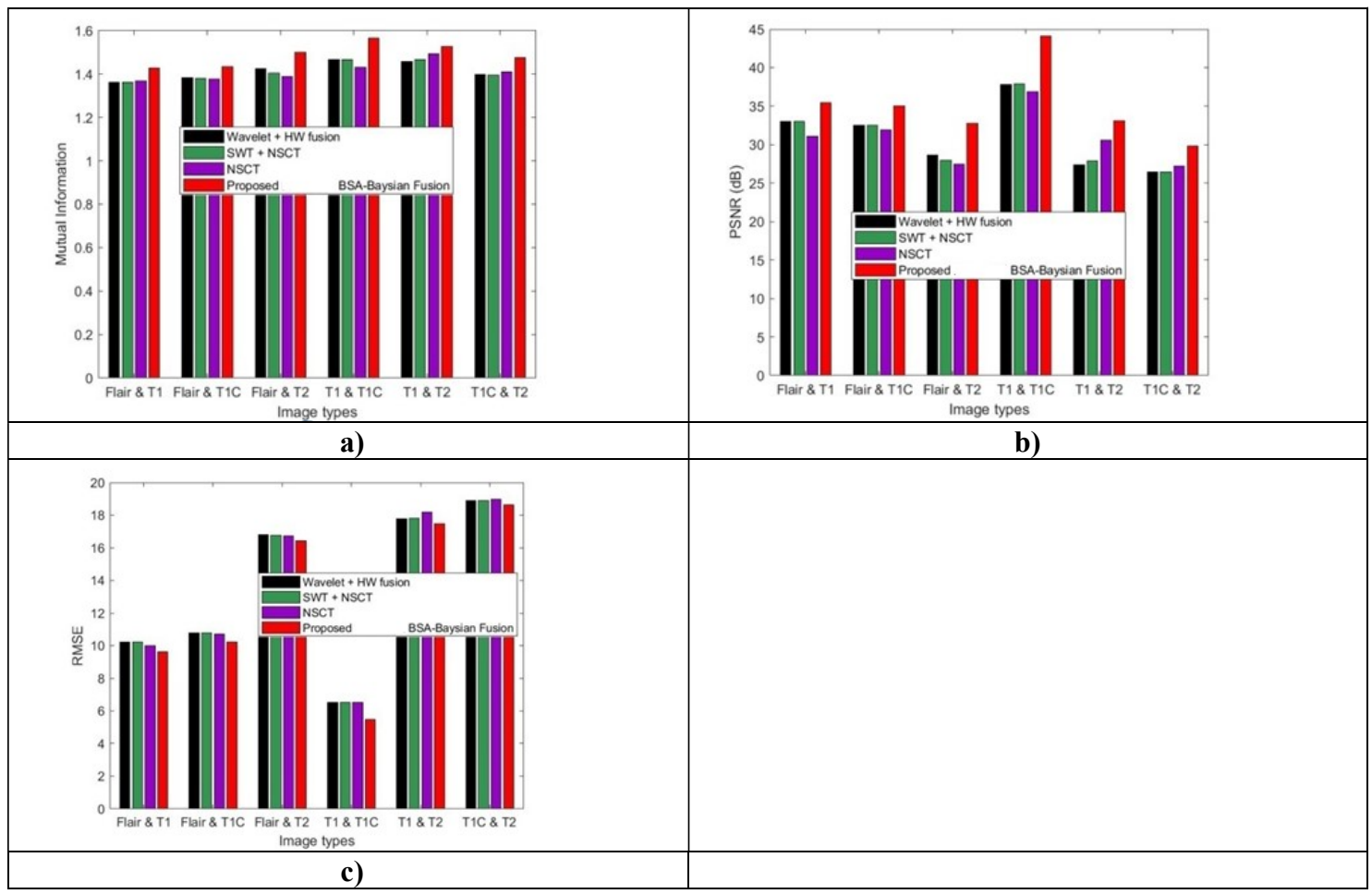

Fig 5: Comparative of Proposed Method with Other Methods, a) Mutual Information, b) PSNR, c) RMSE 


\section{Conclusion}

The image fusion is performed using the MRI brain images taken from the BRATS 2018 database [11], [12] and the images from different modalities in the different set of six are considered for fusion. The six different image sets are Flair\&T1 (Image set-1), Flair\&T1C (Image set-2), Flair\&T2 (Image set-3), T1\&T1C (Image set-4), T1\&T2 (Image set-5) and T1C\&T2 (Image set-6) [11], [12]. Only the results from image set -1 and image set-2 are tabulated and described here with while the graphical results are shown for all six-image sets as shown in Fig.4 and Fig.5. The analysis reveal that the proposed BSA Bayesian fusion outperformed the three recent popular methods of fusion like combined wavelet and Holo entropy - Whale method [2], cascaded framework method of SWT and NSCT [3] and simple NSCT method [4] with a maximal mutual information, maximal PSNR, and minimal RMSE of 1.4764, $37.2114 \mathrm{~dB}$, and 9.9341 respectively for first image data set (Flair and T1) and for second image data set (Flair and T1C) it is $1.4864,37.3114$ and 9.9441 respectively. Every modality has different amount of information. This variation in the result values of all image sets can be analysed in Table 1 and Table 2 as well as in Fig.4 and Fig.5.

\section{Acknowledgements}

This work is supported by University School of Information, Communication and Technology (USICT), Delhi and Bhagwan Parshuram Institute of Technology, Delhi. The authors are also thankful to Dr. Deepak Gambhir, $\mathrm{PhD}$, USICT, Delhi.

\section{References}

[1] Jayant Bhardwaj and Abhijit Nayak,"Lifting Wavelet and KL Transform(LWKL) based CT and MRI Image Fusion Scheme", in Biophotonics Congress: Optics in the Life Sciences Congress), OSA Technical Digest (Optical Society of America), 2019.

[2] Patil Hanmant Venkatrao and Shirbahadurkar Suresh Damodar," HWFusion: Holoentropy and SP-Whale optimisation-based fusion model for magnetic resonance imaging multimodal image fusion", IET Image Processing, Vol: 12, no: 4, pp: 572-581, 2018.

[3] Vikrant Bhateja, Himanshi Patel, Abhinav Krishn, Akanksha Sahu and Aimé Lay-Ekuakille,” Multimodal Medical Image Sensor Fusion Framework Using Cascade of Wavelet And Contourlet Transform Domains", IEEE Sensors Journal, Vol: 15, no: 12, pp: 6783-6790, Dec. 2015.

[4] Gaurav Bhatnagar, Q.M. Jonathan Wu and Zheng Liu,"Directive Contrast Based Multimodal Medical Image Fusion in NSCT Domain", IEEE Transactions on Multimedia, Vol: 15, no:5, Aug., 2013.

[5] Jayant Bhardwaj and Abhijit Nayak, "Cascaded Lifting Wavelet and Contourlet Framework Based Dual Stage Fusion Scheme for Multimodal Medical Images". Journal of Electrical and Electronic System, Dec 2018.

[6] Jayant Bhardwaj and Abhijit Nayak, "Lifting Wavelet Transform based Ultrasound Image Fusion scheme," in Imaging and Applied Optics 2018, OSA Technical Digest (Optical Society of America), 2018.

[7] Sudipta Majumdar and Jayant Bhardwaj, "Feature Level Fusion of Multimodal Images Using Haar Lifting Wavelet Transform", World Academy of Science, Engineering and Technology International Journal of Computer and Information Engineering,Vol:8, no:6, 2014.

[8] Jayant Bhardwaj, Abhijit Nayak, Kulvinder Singh, "Feature Level Fusion of Gray Dentistry Images using Haar Lifting Wavelet Transform," in Imaging and Applied Optics 2017, OSA Technical Digest (online) ,Optical Society of America, 2017.

[9] Yifan Zhang,Steve De Backer and Paul Scheunders, "Noise-Resistant Wavelet-Based Bayesian Fusion of Multispectral and Hyperspectral Images", IEEE Transactions on Geoscience and remote sensing, Vol. 47, No. 11, Nov. 2009.

[10] Xian-Bing Mengab, X.Z. Gaoc, Lihua Lude, Yu Liub \& Hengzhen Zhanga,” A new bio-inspired optimisation algorithm: Bird Swarm Algorithm", Journal of Experimental \& Theoretical Artificial Intelligence, Vol: 28, no: 4, 2016.

[11] Multimodal Brain Imageshttps://www.med.upenn.edu/sbia/brats2018/data.html",accessed, September 2019. [12] B.H.Menze, A. Jakab,S.Bauer, J.Kalpathy-Cramer, K. Farahani, J. Kirby, et al. "The Multimodal Brain Tumor Image Segmentation Benchmark (BRATS)", IEEE Transactions on Medical Imaging 34(10), 1993-2024 ,2015. 